\title{
The inversion relation
}

\author{
J. M. Maillard \\ Laboratoire de Physique Théorique et Hautes Energies, Université Paris VI, Tour 14, ${ }^{\mathrm{er}}$ étage, \\ 4, place Jussieu, 75230 Paris Cedex 05, France
}

(Reçu le 2 juillet 1984, accepté le 20 novembre 1984)

\begin{abstract}
Résumé. - Nous introduisons la notion de relation d'inverse de manière très heuristique (partie I) sur différents exemples simples. Le caractère très général de la relation d'inverse est ainsi souligné. Son importance est ensuite illustrée dans le cadre de calculs analytiques précis (partie II) sur trois classes de problèmes différents : les modèles exactement solubles, le voisinage de la criticité et enfin les modèles (a priori) non solubles pour lesquels un effort est tenté pour caractériser les contraintes découlant de la relation d'inverse.
\end{abstract}

\begin{abstract}
The inversion relation concept is introduced in a very heuristic way (part I) with some simple and different examples. The general character of the inversion relation is emphasized. The importance of the inversion relation is illustrated in the framework of some precise analytical discussions (part II) for three different cases : exactly soluble models, the case of near criticality and a priori non exactly soluble models where an attempt is made to characterize the constraints imposed by the inversion relation.
\end{abstract}

\section{THE INVERSION RELATION : SOME SIMPLE EXAMPLES}

\section{Introduction.}

The inversion relation (IR) has been used in the past few years as a short cut in order to calculate very simply and quickly the partition function of exactly soluble models in statistical mechanics : from this point of view the IR corresponds formally to the unitarity relation on the two-body $S$-matrix in the $1+1$ dimensional $S$ matrix theory; this calculation corresponds to the exact calculation of the $S$-matrix of a $1+1$ dimensional field theory using Watson's equations (unitarity + crossing).

The IR was first introduced in statistical mechanics by Y. G. Stroganov [1] and used very successfully by many authors (Baxter [2], Schultz [3], Pokrovsky and Bashilov [4]...)

It is true that the same results can usually be obtained for the partition function by the use of « classical methods ", the Bethe ansatz (Bethe [5]) but, in the case of the hard-hexagon model for instance, the partition function has been calculated by R. J. Baxter and P. A. Pearce [6] using only the IR and the $\pi / 2$ symmetry rotation of the lattice : no Bethe ansatz for this model is available at the present moment, although there is some evidence that such an ansatz could exist (see Eq. (4.8) of Baxter and Pearce [6]).
The inversion relation is thus a powerful concept within the framework of complete integrability, but it has been shown to exist also outside this framework and to give strong constraints on the partition function : examples are the two-dimensional Ising model in a magnetic field (Baxter [2]), the two-dimensional non-critical Potts model (M. T. Jaekel, J. M. Maillard [7]) for which an inverse functional equation exists for the partition function.

Despite these results on completely integrable or even non-integrable models the IR is not very well known : exact relations are usually referred to in terms of the Kramers-Wannier duality [8] which enables the critical temperature of the two-dimensional Ising model to be determined exactly on a square lattice, and people very often relate this to the fact that the partition function may be calculated exactly (perhaps because of the well known Onsagersolution [9]). In fact we propose to show that the IR is a more powerful and constraining relation than the $\mathrm{K}-\mathrm{W}$ duality, and moreover far more universal than the $\mathrm{K}-\mathrm{W}$ self duality.

The aim of this paper is therefore to popularize the important concept of the IR.

For convenience the paper is divided into two parts headed I and II. Part I gives different simple examples 
of the IR and presents in a very heuristic way that relation. Part II concentrate on exact analytical calculations and analytical discussions of the consequences of the IR.

\section{Inversion relation on quasi dimensional models.}

The inversion relation was introduced mainly to calculate exactly the partition function of two-dimensional models, but is more simply introduced in one-dimensional and quasi one-dimensional models.

1.1 ONE-DIMENSIONAL ISING MODEL IN A MAGNETIC FIELD. - Let us consider one of the simplest lattice models in existence : the one-dimensional Ising model in a magnetic field. We associate with this problem a $2 \times 2$ transfer matrix $T=T_{1} T_{2}$

where

$$
T_{1}=\left(\begin{array}{ll}
\mathrm{e}^{K} & \mathrm{e}^{-K} \\
\mathrm{e}^{-K} & \mathrm{e}^{K}
\end{array}\right)
$$

and

$$
T_{2}=\left(\begin{array}{cc}
\mathrm{e}^{H} & 0 \\
0 & \mathrm{e}^{-H}
\end{array}\right)
$$

( $K$ is a coupling constant, $H$ the magnetic field). Replacing $T$ by $\widetilde{T}=T_{2}^{1 / 2} T_{1} T_{2}^{1 / 2}$, which does not change the partition function of the model with periodic boundary conditions, we find immediately that : $T_{1}(K+i \pi / 2) T_{1}(K)=2 i \operatorname{sh} 2 K .1$ and $T_{2}(H) . T_{2}(-H)$ $=\mathbb{1}$ where $\mathbb{1}$ is the $2 \times 2$ identity matrix, hence $\tilde{T}(K+i \pi / 2, H) . \tilde{T}(K, H)=2 i \operatorname{sh} 2 K$. 1 . If we denote by $\varphi(K, H)$ the sum of the logarithms of the two eigenvalues of the transfer matrix $\widetilde{T}$, we get the equation :

$$
\varphi(K, H)+\varphi(K+i \pi / 2,-H)=2 \ln (2 i \operatorname{sh} 2 K) .
$$

We have already discussed elsewhere [7] the extent to which relation (1) can be said to imply a similar inverse functional equation on the largest eigenvalue of the transfer matrix $\tilde{T}$ (i.e. on the partition function per site denoted by $Z$ )

$$
Z(K, H) . Z(K+i \pi / 2,-H)=2 i \operatorname{sh} 2 K
$$

By taking such a simple example it is possible to check equation (2) directly because the exact expression of $Z$ is known;

$$
Z(K, H)=\mathrm{e}^{K} \operatorname{ch} H+\sqrt{\mathrm{e}^{2 K} \operatorname{sh}^{2} H+\mathrm{e}^{-2 K}} .
$$

We see that equation (2) is actually satisfied if $Z(K+i \pi / 2,-H)$ is chosen as :

$$
\begin{aligned}
& Z(K+i \pi / 2,-H)= \\
& \quad=i \cdot\left[\mathrm{e}^{K} \operatorname{ch} H-\sqrt{\mathrm{e}^{2 K} \operatorname{sh}^{2} H+\mathrm{e}^{-2 K}}\right] .
\end{aligned}
$$

In this precise example we find that $Z(K+i \pi / 2,-H)$ is an analytical continuation of $Z(K, H)$. It is interesting to compare this global analytical approach with a direct verification based on a low-temperature expansion :

$$
\begin{aligned}
Z(K, H)=\mathrm{e}^{K+H} \cdot(1+ & \mathrm{e}^{-4 K} \frac{\mathrm{e}^{-2 H}}{1-\mathrm{e}^{-2 H}}- \\
& \left.-\mathrm{e}^{-8 K} \frac{\mathrm{e}^{-4 H}}{\left(1-\mathrm{e}^{-2 H}\right)^{3}}+\cdots\right) .
\end{aligned}
$$

Such an expansion can be obtained from the resummation of low temperature expansions :
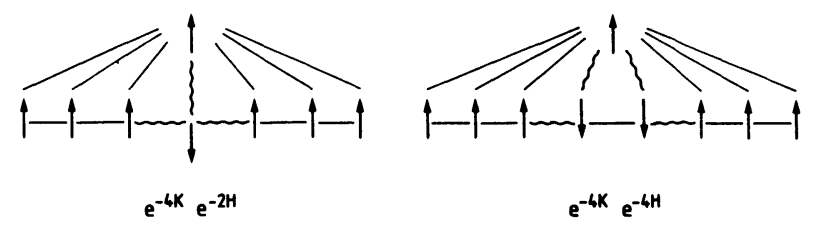

The term $\mathrm{e}^{-4 K} \mathrm{e}^{-2 H}$ corresponds to the reversal of one spin, the term $\mathrm{e}^{-4 K} \mathrm{e}^{-4 H}$ to the reversal of two next nearest neighbour spins... Obviously the sequence of these terms constitutes a geometric series of which the associated contribution is $\mathrm{e}^{-4 K} \frac{\mathrm{e}^{-2 H}}{1-\mathrm{e}^{-2 H}}$; this is also the expansion of (3) for small $\mathrm{e}^{-K}$. It is easy to check (3) at the lowest order :

$$
\begin{aligned}
& \mathrm{e}^{K+H}\left(1+\mathrm{e}^{-4 K} \frac{\mathrm{e}^{-2 H}}{1-\mathrm{e}^{-2 H}}+\cdots\right) \times \\
& \times \mathrm{e}^{(K+i / 2)-H}\left(1+\mathrm{e}^{-4 K} \frac{\mathrm{e}^{2 H}}{1-\mathrm{e}^{2 H}}+\cdots\right)= \\
& =i \mathrm{e}^{2 K}\left(1-\mathrm{e}^{-4 K}\right) .
\end{aligned}
$$

The second term in the product of equation (5) is actually the expansion of (4) for small $\mathrm{e}^{-\mathrm{K}}$.

The one-dimensional Ising model in a magnetic field is a simple model satisfying other exact properties such as the existence of a Kramers-Wannier self duality and that of a star-triangle relation, which generates an exact renormalization group for this model. It can be verified that both these exact properties are compatible with the inversion relation (2).

1.2 Rubber BANDS : THE ANISOTROPIC PotTS MOdEL. - Let us consider the Potts model on a simple rubber band.

In the case of a Potts model, $\sigma_{i}$ spins on the vertex of the lattice belong to $Z_{q}$; therefore if $N$ denotes the size of the strip, the transfer matrices $T_{1}, T_{2}$ and $\tilde{T}$ corresponding to this model are $q^{N} \times q^{N}$ matrices : these matrices are in general too large to allow any practical analytical calculation, but fortunately they split into matrices of smaller size (Temperley and Lieb [10], Blöte et al. [11]) (their size grows as a 
polynomial in $N$, independently of $q$ ). For instance, in the $N=3$ case, the largest eigenvalue (partition function) is that of a $3 \times 3$ matrix denoted by $t$, which is the product of two $3 \times 3$ matrices $t_{1}$ and $t_{2}$ dependent respectively on $K_{1}$ and $K_{2}$, the vertical and horizontal coupling constants of anisotropic Potts model. The exact expressions of $t_{1}\left(K_{1}\right)$ and $t_{2}\left(K_{2}\right)$ are given in the appendix in the case of transversal periodic boundary conditions. It is quite easy to verify that

$$
\begin{gathered}
t_{2}\left(K_{2}\right) \cdot t_{2}\left(-K_{2}\right)=e \\
t_{1}\left(K_{1}\right) \cdot t_{1}\left(\left(-K_{1}^{*}\right)^{*}\right)=e
\end{gathered}
$$

where $e$ refers to the $3 \times 3$ identity matrix and the star to the duality transformation for the Potts model. This result may also be derived from the observation that matrices $t_{1}$ and $t_{2}$ are simple exponentials of a constant matrix (see Appendix). This exponentiation property is reminiscent of the situation in the twodimensional Ising (Kasteleyn [12]) and even Potts model (Stephen and Mittag [13]). Given equations (6) and (7), and introducing the symmetrized matrix $\tilde{t}=t_{1}^{\frac{1}{2}} t_{2} t_{1}^{\frac{1}{2}}$ we get :

$$
\tilde{t}\left(K_{1}, K_{2}\right) \cdot \tilde{t}\left(\left(-K_{1}^{*}\right)^{*},-K_{2}\right)=e .
$$

Arguing from equation (8) we have (introducing a correct multiplicative factor and the notations $\left.b=\mathrm{e}^{K_{1}}, c=\mathrm{e}^{K_{2}}\right)$ :

$$
Z(b, c) \cdot Z\left(2-q-b, \frac{1}{c}\right)=(b-1)(1-q-b)
$$

$Z(b, c)$ corresponds to the largest eigenvalue of $\tilde{t}\left(K_{1}, K_{2}\right)$ and $Z\left(2-q-b, \frac{1}{c}\right)$ is an analytical continuation of $Z(b, c)$ (one of the three eigenvalues of $\left.t\left(\left(-K_{1}^{*}\right)^{*},-K_{2}\right)\right)$. Of course it is possible to check equation (9) by introducing a resummed high (or low) temperature expansion

$$
\begin{aligned}
& \left(b^{*}=\frac{b+q-1}{b-1}, \quad c^{*}=\frac{c+q-1}{c-1}\right) \\
& \square(q-1) \frac{1}{c^{* 2}} \frac{1}{b^{* 2}-1} \\
& \square(q-1)(q-2) \frac{1}{c^{* 3}}\left(\frac{1}{b^{* 2}-1}\right)^{2} .
\end{aligned}
$$

This resummed expansion up to the fifth order in $1 / c^{*}$ has been given elsewhere [7] and enables us to check to the same order that equation (9) is indeed verified. Let us take only the third order : introducing the normalized partition function $\Lambda(b, c)$, which tends to 1 in the high temperature limit, equation (9) can be rewritten

$$
\begin{aligned}
\ln \Lambda(b, c)+\ln \Lambda(2 & \left.-q-b, \frac{1}{c}\right)= \\
= & \ln \left(\frac{(c-1)(1-q-c)}{c(2-q-c)}\right)
\end{aligned}
$$

$$
\begin{aligned}
\ln \Lambda(b, c)= & (q-1) \frac{1}{c^{* 2}} \frac{1}{b^{* 2}-1}+ \\
& +(q-1)(q-2) \frac{1}{c^{* 3}}\left(\frac{1}{b^{* 2}-1}\right)^{2}+\cdots
\end{aligned}
$$

Equation (10) is actually verified. It should be noted that, as in the Ising case, up to the fifth order in $1 / c^{*}$ the resummed high temperature expansion is the same for the strip of size $N=3$ and for the two-dimensional Potts model.

\section{Inversion relation on two-dimensional models.}

The importance of the IR has appeared mainly in the case of two-dimensional models as illustrated by examples of the two-dimensional anisotropic Ising model (Baxter [2]), some vertex models (Schultz [3]), the hard-hexagon model (Baxter, Pearce [6]) and of course the symmetrical eight-vertex model, also called the Baxter model (Baxter [15] and [16]). It is possible of course to check the associated inverse functional equation directly on the exactly known analytical expressions of the partition function for these models. On the other hand we shall see in paper II that from the IR and some obvious symmetry considerations we get very easily the exact expression of the partition function. Therefore, instead of recalling a direct verification of the IR, we shall check it on the Baxter model via the resummed high-temperature expansion to the lowest orders.

2.1 INVERSION RELATION IN THE CASE OF THE IRF MODEL. - Let us consider the very general case of the interaction round-a-face model (IRF model) (Baxter [2]). In this model, to each plaquette of a square lattice is assigned the most general Boltzmann weight $W$, dependent on the $2^{4}$ configurations of the four Ising spins at the four corners of each plaquette

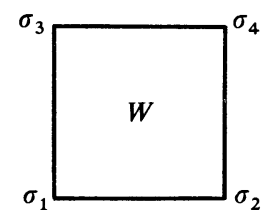

This model therefore has sixteen parameters. Let us give a definition and a graphical representation of the IR for this model : the IR implies that the partition 
functions of the two graphs below are equal

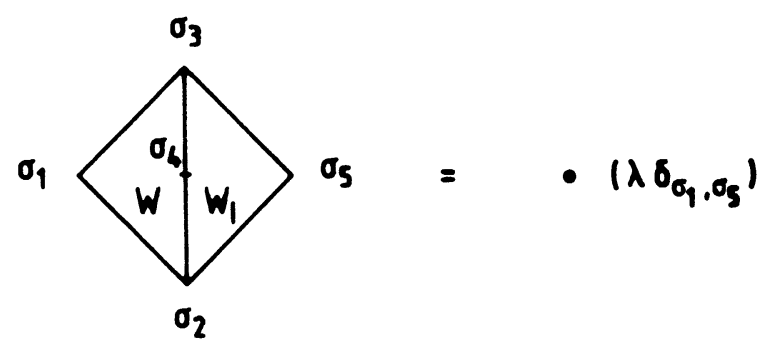

Fig. 1.

which in analytical terms means that for all $\sigma_{2}$ and $\sigma_{3}$ :

$$
\sum_{\sigma_{4}} W\left(\sigma_{1}, \sigma_{2}, \sigma_{4}, \sigma_{3}\right) . W_{\mathrm{I}}\left(\sigma_{4}, \sigma_{2}, \sigma_{5}, \sigma_{3}\right)=\lambda . \delta_{\sigma_{1}, \sigma_{5}}
$$

( $\lambda$ is some known expression, $\delta$ the usual Kronecker delta symbol).

Let us now consider figure 2, which represents the product of the two horizontal transfer matrices (with periodic boundary conditions) $T(W)$ and $T\left(W_{1}\right)$ associated with the Boltzmann weight $W$ and its « inverse » Boltzmann weight $W_{1}$ respectively :

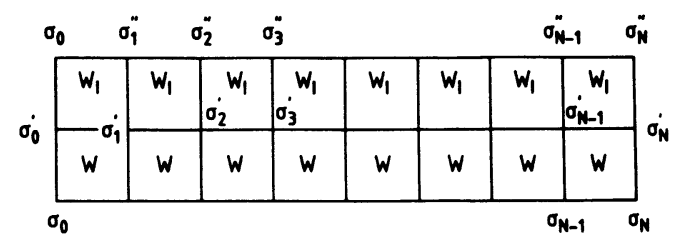

$\sigma_{0}^{*}=\sigma_{N}^{*}$ $\dot{\sigma}_{0}=\dot{\sigma}_{N}$

$\sigma_{0}=\sigma_{\mathrm{N}}$

Fig. 2.

Let us assume that one $\sigma_{i}$ at least is in the same state as $\sigma_{i}^{\prime \prime}$ : because of this identification we locally recover figure 1 ; using the very definition of the inversion relation we find that $\sigma_{i+1}^{\prime \prime}$ and $\sigma_{i+1}$ must be in the same state and therefore, iterating this procedure, that every $\sigma_{k}$ has to identify with its corresponding $\sigma_{k}^{\prime \prime}$. In other words the product of the two $2^{N+1} \times 2^{N+1}$ transfer matrices $T(W)$ and $T\left(W_{\mathrm{I}}\right)$ is equal to $\lambda^{N} \cdot 1+M$, where 1 is the $2^{N+1} \times 2^{N+1}$ identity matrix and $M$ a matrix of which the coefficients are non zero only when $\sigma_{k}=-\sigma_{k}^{\prime \prime}$ for every $k$. We shall not try to argue here that within the thermodynamic limit this additional matrix $M$ is negligible. Such a demonstration must only be considered as a heuristic argument enabling the following inverse functional equation to be written for the partition function : $Z(W) \cdot Z\left(W_{\mathrm{1}}\right)=\lambda(W)$, this equation being checked by diagrammatic expansions or any other method. We should merely note that, in the case of vertex models, introducing the diagonal transfer matrix with periodic boundary conditions, we get, for the product $T(W) T\left(W_{\mathrm{I}}\right)$, a result rigorously proportional to the identity matrix without the involvement of any correction $M$ to neglect.

2.2 INVERSION RELATION ON THE BAXTER MODEL. The Baxter model $[15,16]$ is a particular case of the IRF model :

$$
W\left(\sigma_{1}, \sigma_{2}, \sigma_{3}, \sigma_{4}\right)=\mathrm{e}^{K_{1} \sigma_{1} \sigma_{4}+K_{2} \sigma_{2} \sigma_{3}+M \sigma_{1} \sigma_{2} \sigma_{3} \sigma_{4}} .
$$

It is easily verified that the inverse Boltzmann weight $W_{1}$ corresponds to the new parameters $K_{1}^{\prime}, K_{2}^{\prime}, M^{\prime}$ defined by $K_{1}^{\prime}=K_{1}+i \pi / 2, M^{\prime}=M$ and

$$
\mathrm{e}^{2 K_{2}^{\prime}}=\mathrm{e}^{-2 K_{2}} \cdot \frac{\operatorname{sh}\left(2 K_{1}-2 M\right)}{\operatorname{sh}\left(2 K_{1}+2 M\right)}
$$

$\lambda(W)$ is equal to $2 i\left(\operatorname{sh}\left(2 K_{1}-2 M\right)\right)\left(\operatorname{sh}\left(2 K_{1}+2 M\right)\right)^{\frac{1}{2}}$. For $M=0$ we recover the IR for the two-dimensional anisotropic Ising model. Let us verify, to the lowest order the inverse functional relation

$$
\begin{aligned}
& Z\left(K_{1}, K_{2}, M\right) \cdot Z\left(K_{1}+i \pi / 2, K_{2}^{\prime}, M\right)= \\
& =2 i\left(\operatorname{sh}\left(2 K_{1}-2 M\right) \operatorname{sh}\left(2 K_{1}+2 M\right)\right)^{\frac{1}{2}}
\end{aligned}
$$

or if we introduce the high temperature normalized partition function

$$
\begin{aligned}
& \Lambda\left(K_{1}, K_{2}, M\right)= \\
& =Z\left(K_{1}, K_{2}, M\right)\left(\operatorname{ch} K_{1} \operatorname{ch} K_{2} \operatorname{ch} M\right)^{-1} \\
& \ln \Lambda\left(K_{1}, K_{2}, M\right)+\ln \Lambda\left(K_{1}+i \pi / 2, K_{2}^{\prime}, M\right)= \\
& =\ln \left[\frac{\left(\operatorname{sh}\left(2 K_{1}-2 M\right) \operatorname{sh}\left(2 K_{1}+2 M\right)\right)^{\frac{1}{2}}}{\operatorname{sh} 2 K_{1}}\right]- \\
& -\ln \left(\operatorname{ch} K_{2} \operatorname{sh} K_{2}^{\prime} \operatorname{ch}^{2} M\right)
\end{aligned}
$$

As in the case of the Ising model we introduce the high-temperature variables :

$$
\begin{gathered}
t_{1}=\text { th } K_{1}, \quad t_{2}=\text { th } K_{2}, \quad t=\text { th } M, \\
t_{2}^{\prime}=\text { th } K_{2}^{\prime}=-\frac{\left[t\left(1+t_{1}^{2}\right)+t_{1}\left(1+t^{2}\right) t_{2}\right]}{\left[t_{1}\left(1+t^{2}\right)+t\left(1+t_{1}^{2}\right) t_{2}\right]} .
\end{gathered}
$$

The expansion of the right hand side of equation (15) gives at the lowest order in $t$ and $t_{2}$ :

$$
\begin{aligned}
-t^{2}-t_{2}^{2}-\left(t_{1}+\frac{1}{t_{1}}\right) \cdot t t_{2}- & \frac{1}{2}\left(t_{1}+\frac{1}{t_{1}}\right)^{2} \cdot t^{2}- \\
& -\frac{t^{2}}{2}\left(t_{1}-\frac{1}{t_{1}}\right)^{2}+\cdots
\end{aligned}
$$

If we denote by a bubble the th $M$ term in the high temperature diagrams we get the following diagrams (and associated contributions) at the lowest order in $t$ 
and $t_{2}$ :

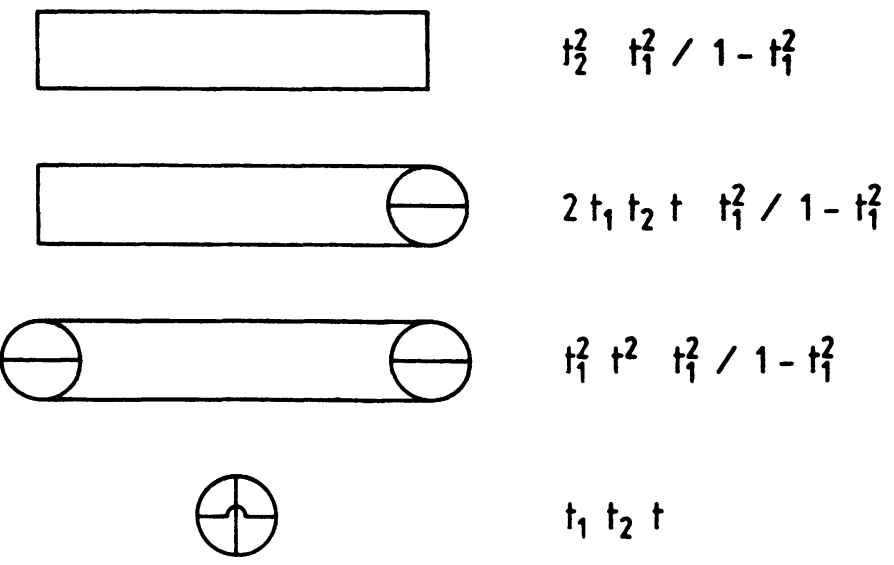

Fig. 3.

The verification of equation (15) is a straightforward exercise : we obviously recover the $t_{2}^{2}$ term (this is the Ising $M=0$ verification) and also the terms $-t t_{2}\left(t_{1}+\frac{1}{t_{1}}\right)$ and $-t^{2}\left(t_{1}^{2}+1+\frac{1}{t_{1}^{2}}\right)$ using the expansion of $t_{2}^{\prime}$.

\section{Inversion relation on models not known to be exactly solvable : universal nature of the IR.}

An inverse functional equation has been obtained for almost all exactly solvable models but it must be recalled that such an equation also exists for some important two-dimensional models such as the anisotropic Ising model in a magnetic field (Baxter [2]) and the non-critical Potts model (Jaekel and Maillard [7]) which seem not to be solvable. An inverse functional equation also exists for higher-dimensional models such as for example the anisotropic threedimensional Ising model (Jaekel and Maillard [17]). These three a priori non exactly solvable examples refer to important and natural models in statistical mechanics; they also show that the IR can exist outside the framework of complete integrability or, more specifically that it is possible to find an IR symmetry on a model where no star-triangle relation has been found at the present time. This opens up a new and, as we shall see, large class of models which satisfy simple and constraining equations on the partition function but are not at first sight completely integrable. The connection between the IR and the star triangle relation has been studied elsewhere (Garel and Maillard [18]).

The anisotropic two-dimensional Ising model in a magnetic field and the anisotropic three-dimensional Ising model are not self-dual models. Thus taking the K-W dual of these two models we get two Gauge models on a lattice and two inverse functional equations for these two Gauge models which are the images by the dual transformation of the preceding inverse functional equations. Let $\lambda_{i j}$ denote Ising variables associated with the link $\langle i j\rangle, \prod_{\langle i j\rangle}\left(\right.$ resp. $\left.\prod_{\langle j k\rangle}\right)$ the product over all horizontal (resp. vertical) bonds on the lattice, and also $\prod_{\langle\rho\rangle}$ the product over all plaquettes on the lattice.

The partition function of the dual model of the two-dimensional Ising model with a magnetic field is

$$
Z=\sum_{\{\lambda\}} \prod_{\langle i j\rangle} \mathrm{e}^{K_{1} \lambda_{i j}} \prod_{\langle j k\rangle} \mathrm{e}^{K_{2} \lambda_{j k}} \prod_{\langle\rho\rangle} \mathrm{e}^{K \lambda_{i j} \lambda_{j k} \lambda_{k l} \lambda_{l i}} .
$$

The inverse functional relation for that Gauge model is

$$
\begin{aligned}
Z\left(K_{1}, K_{2}, K\right) . Z\left(-K_{1}, K_{2}\right. & +i \pi / 2, K+i \pi / 2)= \\
& =2 i \operatorname{sh} 2 K_{2} \cdot 2 i \operatorname{sh} 2 K .
\end{aligned}
$$

Similarly the dual of the three-dimensional Ising model is a pure Gauge model where, instead of one, we have three plaquette coupling constants $K_{1}, K_{2}, K_{3}$ (corresponding to the three axes of the cubic lattice) and the inverse functional relation for this pure gauge model is

$$
\begin{array}{r}
Z\left(K_{1}, K_{2}, K_{3}\right) \cdot Z\left(-K_{1}, K_{2}+i \pi / 2, K_{3}+i \pi / 2\right)= \\
=2 i \operatorname{sh} 2 K_{2} .2 i \operatorname{sh} 2 K_{3} .
\end{array}
$$

It is possible to find many other models for which an inverse functional relation exists (many body interactions, interaction between next nearest neighbours, Potts models), but it is clear that the IR becomes less relevant if the models depend on too many parameters. One should observe that a self-duality symmetry exists for fewer models than does an IR. Moreover the self-duality symmetry is less constrain- 
ing than the inversion relation, which, combined with the symmetry relation, leads to an infinite discrete group. The study of that infinite group $G$ is made in paper (II) in the case of the 2-d Potts model.

Therefore the partition function has an infinite symmetry group which, as will be recalled in paper (II), enables us, among many other consequences, to recover the critical manifold obtained from the duality relation but also to determine critical manifolds that cannot be obtained from the duality relation. Thus, the IR imposes stronger restraints on the model than the duality transformation; the reason for this is that the duality transformation commutes with all the elements of that infinite group $G$ (because it commutes with the inversion and symmetry relations). This compatibility can be understood from a general point of view (Maillard [14]). Hence, combining the duality transformation with the IR or the symmetry relation, we get a finite number of elements. This is definitively not enough in the case of anisotropic models.

Let us add a final word on the general character of the inversion relation : the IR implies a functional equation not only for the largest eigenvalue (the partition function) but also for other eigenvalues of the transfer matrix. This can be checked on different models : the symmetrical six-vertex model, the Baxter model, or the two-dimensional Ising model (Jaekel and Maillard [7], Maillard [19]). The inversion relation also leads to a functional equation in the case of the n-point correlation function (Jaekel and Maillard [20]), as well as for different quantities such as magnetization or susceptibility. For instance, by writing the IR on the anisotropic two-dimensional Ising model in a magnetic field, and taking the second partial derivative with respect to the magnetic field, we get the equation for the susceptibility $\chi$ :

$$
\chi\left(K_{1}, K_{2}\right)+\chi\left(K_{1}+i \pi / 2,-K_{2}\right)=0 .
$$

\section{Conclusion.}

The star-triangle has been recognized by many authors as a key point in the understanding of exact models. We recall in this paper that the inversion relation exists even in the absence of a star-triangle relation.

The inversion relation, although somewhat similar superficially to the Kramers-Wannier duality, is more constraining. Moreover such a relation exists for a large number of models with no K-W selfduality (the three-dimensional Ising model for instance).

The large set of models for which the inversion relation exists can be shown to include : quasi onedimensional models (rubber bands), two-dimensional models with or without magnetic field on different lattices (even non-planar) or with four-spin interactions, and three-dimensional models like the Ising or Potts cubic model (possibly with magnetic field).

In fact this list is far from exhaustive and many other examples can be found. The IR enables simple functional equations to be written for the partition function, but also for other quantities such as the correlation function, interface energy, magnetization, and so on.

The field of application of the IR therefore seems quite broad, a fact which is not in contradiction with the relevant character of these equations as will be emphasized in a second paper.

\section{Appendix}

Rubber BANDS : THE ANISOTROPIC PotTS MODEL. tions :

Analytic expression for $t_{1}$ and $t_{2}$; let $u$ denote $\mathrm{e}^{K_{2}}-1$ and $u^{\prime}, \mathrm{e}^{K_{1}}-1$ and $\mathrm{e}^{K_{1}}-1=q / u^{\prime *}$; with these nota-

$$
\left.\begin{array}{l}
t_{2}(u)=\left(\begin{array}{ccc}
1 & 0 & 0 \\
+\frac{3 u}{q} & 1+u & 0 \\
\frac{3 u^{2}+u^{3}}{q^{2}} & \frac{(1+u)\left(2 u+u^{2}\right)}{q} & (1+u)^{3}
\end{array}\right)=\exp \left[K_{2}\left(\begin{array}{ccc}
0 & 0 & 0 \\
3 / q & 1 & 0 \\
0 & 2 / q & 3
\end{array}\right)\right.
\end{array}\right] .
$$


Denoting by $R$ the matrix $\left(\begin{array}{ccc}0 & 0 & q \\ 0 & 1 & 0 \\ q^{-1} & 0 & 0\end{array}\right)$, one has the following relation between $t_{1}$ and $t_{2}$ :

$$
\left(u^{\prime *}\right)^{3} \cdot R t_{1}\left(u^{\prime}\right) R=t_{2}\left(u^{*}\right)
$$

\section{References}

[1] Stroganov, Y., Phys. Lett. 74A (1979) 116.

[2] BAXTER, R. J., Fundamental problems in statistical mechanics $V$; Proceedings of the 1980 Enschede Summer School; E. G. D. Cohen ed. (NorthHolland Amsterdam) 1980.

[3] Schultz, C. L., Phys. Rev. Lett. 46 (1981) 629.

[4] Pokrowsky, S. V., Bashilov, Y. A., Comm. Math. Phys. 84 (1982) 103.

[5] Bethe, H. A., Z. Phys. 71 (1931) 205.

[6] Baxter, R. J., Pearce, P. A., J. Phys. A 15 (1982) 897.

[7] Jaekel, M. T., Maillard, J. M., J. Phys. A 15 (1982) 2241-2257.

[8] Kramers, H. A., Wannier, G. H., Phys. Rev. 60 (1941) 252.

[9] ONSAGER, L., Phys. Rev. 65 (1944) 117.

[10] Temperley, H. N. V., Lieb, E. M., Proc. R. Soc. A 322 (1971) 251.

[11] Blöte, H. W. J., Nightingale, M. P., Derrida, B., J. Phys. A 14 (1981) L 45.
[12] KaSteleyn, P. W., Fundamental problems in Statistical Mechanics III ; Proceedings of the 1975 Wageningen Summer School; E. G. D. Cohen ed., (North-Holland Amsterdam) 1975.

[13] Stephen, M. J., Mittag, L., J. Math. Phys. 13 (1972) 1944.

[14] Maillard, J. M., Note CEA N-2327 (1983).

[15] Baxter, R. J., Ann. Phys. 70 (1972) 193-228.

[16] Baxter, R. J., Ann. Phys. 71 (1973) 1.

[17] Jaekel, M. T., Maillard, J. M., J. Phys. A 15 (1982) 1309-1325.

[18] Garel, T., Maillard, J. M., J. Phys. A 16 (1983) 2257-2265.

[19] Maillard, J. M., Brasov International Summer School; Birkhaüser ed. (1984).

[20] Jaekel, M. T., Maillard, J. M., J. Phys. A 16 (1983) 1975. 